\title{
Adherence to vector preventive measures decrease cases of acute Dengue among Abuja residents, Nigeria
}

\author{
Idris Abdullahi Nasir, ${ }^{1,2}$ Adamu Babayo, ${ }^{3}$ Muhammad Sagir Shehu, ${ }^{4}$ Peter Omale Musa ${ }^{4}$ \\ ${ }^{1}$ Department of Medical Laboratory Services, University of Abuja Teaching Hospital, Gwagwalada; ${ }^{2}$ Department of \\ Medical Microbiology and Parasitology, College of Health Sciences, University of Ilorin; ${ }^{3}$ Department of Medical \\ Microbiology and Parasitology, Faculty of Clinical Sciences, Bayero University, Kano; ${ }^{4}$ Department of Medicine, \\ Ahmadu Bello University, Nigeria
}

\begin{abstract}
Summary
Background: Nigeria is one of the dengue hyper-endemic nations. This study investigated the level of knowledge about dengue and vector preventive practices and their impacts on acute dengue among febrile patients at Abuja, Nigeria.

Materials and methods: This cross-sectional survey was conducted on febrile patients attending University of Abuja Teaching Hospital, Abuja, Nigeria. Blood samples were individually collected from 171 febrile patients residing at Gwagwalada suburb. Interviewer administered questionnaires were used to assess subjects' knowledge about preventive measures against vector breed-
\end{abstract}

Correspondence: Idris Abdullahi Nasir, Department of Medical Laboratory Services, University of Abuja Teaching Hospital, PMB 228 Gwagwalada, FCT Abuja, Nigeria.

Tel.: +234.8022.851352.

E-mail: eedris888@yahoo.com

Key words: Risk factors; Prevention; Dengue; Vector.

Acknowledgements: We greatly appreciate Dr. Olajide Agbede, University of Ilorin, Nigeria for his technical input towards the success of this study.

Contributions: IAN conceptualised the study design and conducted the study as well as developed the initial draft manuscript; $\mathrm{AB}$, gave input into the design and statistical aspects of the study, and critically revised the manuscript before publication; POM and MSS provided guidance on study design and development of the study; all authors read and approved the final manuscript.

Conflict of interest: the authors declare no potential conflict of interest.

Received for publication: 16 March 2017.

Revision received: 26 April 2017.

Accepted for publication: 26 April 2017.

CCopyright I.A. Nasir et al., 2017

Licensee PAGEPress, Italy

Microbiologia Medica 2017; 32:6704

doi:10.4081/mm.2017.6704

This article is distributed under the terms of the Creative Commons Attribution Noncommercial License (by-nc 4.0) which permits any noncommercial use, distribution, and reproduction in any medium, provided the original author(s) and source are credited. ing and bites. Blood samples were tested for dengue virus Nonstructural glycoprotein-1antigen using enzyme linked Immunosorbent assay (ELISA).

Results: Fifteen out of 171 febrile persons (8.8\%) were Dengue NS1 positive. Sixty percent of the subjects do not know about dengue, while 33\% knew about dengue virus infection through television/ radio programs, 5\% through healthcare professionals and $2 \%$ from friends/families. Those who persistently use indoor residual spraying and long sleeves/trousers during daytime had less cases of DENV NS1 than those who do not. There was statistical association between DENV NS1 and residence in proximity to waste dumpsites $(\mathrm{P}<0.0001)$ and frequent use of long sleeve clothing and trousers $(\mathrm{P}=0.005)$. However, there was no statistical association between DENV NS1 antigenemia and persistent use of indoor residual spraying and presence of in-door water containers $(\mathrm{P}>0.05)$.

Conclusions: Findings from this study imply that proper education and adherence to preventive measures minimize people from being susceptible to Dengue virus infections.

\section{Introduction}

Dengue is reemerging and expanding in several countries even those previously unaffected. It has become one of the worst mosquito-borne viral infection of last 2 decades. Dengue and its vectors are widely distributed throughout tropical and subtropical countries including Nigeria (1). Effective vector preventive and control measures are essential strategic direction to reduce Dengue associated morbidity and mortality (1). The main arthropod vector for transmission of dengue virus is Aedes aegypti (2). In addition, Aedes albopictus, Aedes polynesiensis and Aedes furcifer have been shown to be alternative vectors for dengue transmission (3). In Abuja, only Aedes aegypti have been reported to exist (4). Importantly, Aedes spp of mosquitoes are particularly active during the daytime; this makes it very difficult in controlling them $(3,4)$. The World health Organization considered dengue as neglected topical disease, based on its historical lack of coordinated efforts such as political will, and research attention despite the significant mortality, morbidity and socioeconomic burden it places globally $(3,4)$. In view of these, dengue has been prioritized by the WHO through Global Strategy for Dengue Prevention and Control, 2012-2020 (3, 4).

Sustainable vector control is one technical aspects of the Global Strategy for Dengue Prevention and Control. Considering the limited dengue therapeutic modalities and unavailability of 
vaccine in most endemic countries, effective vector control measures are essential strategic component to reduce dengue mortality and morbidity $(3,5)$. Residence in proximity to waste dumpsites has been an environmental determinant and risk of Dengue virus infection. Vectors of Dengue virus in the urban cycle lay eggs in artificial water containers that could be found in many homes and waste dumpsites $(5,6)$. Presence of artificial water containers in homes and streets may favour vector population, expansion and invariably places people who reside in such communities at high risk of contracting Dengue (6).

Because of its close similarity of dengue associated symptoms with other fever-causing infections such as malaria, typhoid and viral hepatitis, an affordable, time-saving and convenient diagnostic test for confirming dengue is needful. It was recently established that dengue virus non-structural protein 1 (NS1) can be detected in the peripheral blood from the first day after susceptible persons are bitten by the infected Aedes mosquitoes. The detection of NS1 offers a larger window of opportunity for diagnosis of acute dengue; this is particularly useful in clinical care, public health and epidemiological purposes (6).

In light of the potential for continued expansion of dengue globally, this study was instigated to determine the impact of knowledge and practice of available preventive measures on the occurrence of acute dengue among people living in Gwagwalada suburb, FCT Abuja, Nigeria.

\section{Materials and Methods}

After ethical approval from the University of Abuja Teaching Hospital, blood samples were individually collected from 171 consented febrile patients residing at Gwagwalalada suburb, an outskirt of Federal Capital territory Abuja, Nigeria. Thereafter, interviewer administered questionnaires were used to assess subjects' knowledge, clinical signs/symptoms of illness, attitude and preventive measures against vector breeding and bites. These questionnaires included the following sections: Do you know about dengue?, How did you know about dengue?, What is its mode of transmission?, What are the signs of ill-health do you experience?, Where do you reside?, Any refuse dumpsite, hollow water collection proximal ( $<50$ meters) to your residence?, Do you frequently wear long sleeve shirts and long trousers?, Do you frequently (daily) use indoor residual spraying?

\section{Dengue NS1 enzyme linked immunosorbent assay}

Sera from $3 \mathrm{ml}$ of 171 blood samples collected from subjects were separated by allowing the blood to clot at room temperature and then spun at $2500 \mathrm{rpm}$ for 15 minutes. Separated sera were labelled with corresponding subject's identification number. Thereafter, sera samples were tested for dengue virus NS1 using Euroimmun ${ }^{\circledR}$ IgG ELISA Kit (Lübeck, Germany). Based on kit manufacturer's manual, the DENV NS1 assay uses amplified twostep sandwich enzyme linked immunosorbent assay to detect and quantify the concentrations of NS1 protein in serum samples. The wells of each microplate have been coated with a highly effective monoclonal anti-Dengue NS1 antibody. The antibodies are specific to all of the Dengue viruses serotypes. The presence of the NS1 antigen is assessed by spectrophotometric (450 nanometers) absorbance obtained using an enzyme-conjugate-HRP and liquid TMB (3,3',5,5'-tetramethylbenzidine) substrate. A set of negative, positive and cut-off controls were provided as internal quality control in order to monitor the functional integrity of the kit.

\section{Statistical analysis}

Results generated from laboratory analysis and data compiled from the questionnaire were analysed using Statistical Package for Social Sciences (SPSS) version 20 (IBM, California Inc. USA). Chi square test was used to determine the level of significance in the occurrence of dengue virus NS1 in association with the various preventive measures adopted by subjects at confidence interval of $95 \%$. P-values were reported to be statistically significant at $<0.05$.

\section{Results}

The age of all subjects ranged from 1 to 71 years. The mean age was 28.6 years, with median age of 23 years and standard deviation of 15 years (Table 1). Fifteen out of 171 febrile persons $(8.8 \%)$ were Dengue NS1 positive. Sixty percent of the subjects do not know about dengue, while 33\% knew about dengue virus infection through television/ radio programs, 5\% through healthcare professionals and $2 \%$ from friends/families (Figure 1). Those who persistently use of indoor residual spraying and long sleeves/ trousers during daytime had less cases of DENV NS1 than those who do not (Table 2). There was statistical association between DENV NS1 antigenemia and residence in proximity ( $<50$ meters) to waste dumpsites $(\mathrm{P}<0.0001)$ and frequent use of long sleeve clothing and trousers $(\mathrm{P}=0.005)$. However, there was no statistical association between DENV NS1 antigenemia and persistent use of indoor residual spray and presence of in-door water containers $(\mathrm{P}>0.05)$. Based on clinical signs of illness 15 subjects with DENV NS1 antigenemia had fever (7.8\%). Out of these, $6(9.5 \%)$ had only fever as principal symptom. In the remaining patients a combination of symptoms were present had fever and headache (15.6\%), fever and skin rash (12.5\%) and 5.0\% had more than 2 clinical signs. None of these subjects with DENV NS1 antigenemia had abdominal, muscle and joint pain. There was no statistical association between NS1 antigenemia and clinical signs of illness $(\mathrm{P}>0.05)$ (Table 3).

\section{Discussion}

Over $75 \%$ of Gwagwalada residents live in very overcrowded areas while about $20 \%$ live deep within the villages, and only $5 \%$

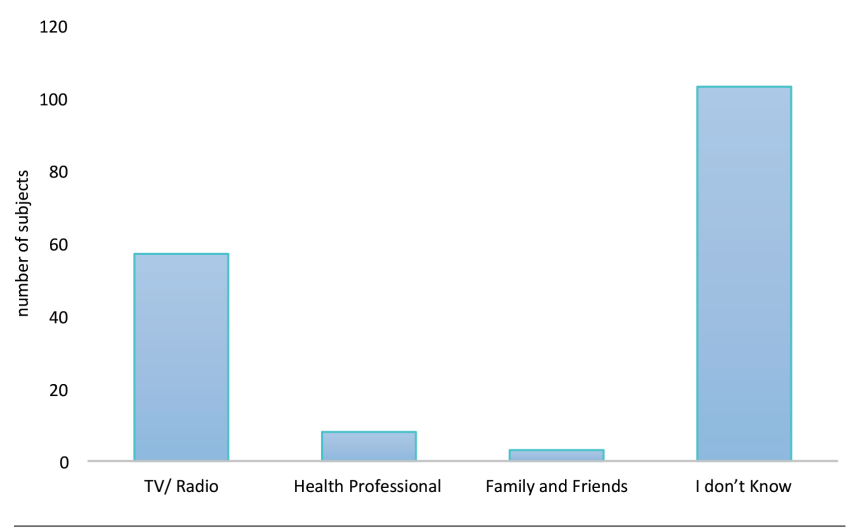

Figure 1. Subjects' source of information about dengue virus infection. 
are found in or close to urban areas (7). Population growth and poor environmental hygienic conditions could be a major factor that encourages dengue virus expansion in Gwagwalada, because in the last five years, this suburb has experience inflow of several settlers because of its relatively lower cost of living and proximity to federal road that links major northern and southern cities of the Nigeria.

The participants in this study had low knowledge of dengue virus. However, most of the participants were aware that dengue is transmitted through mosquito bite(s). This is in accordance to studies conducted in Malaysia and Jamaica, where participants could identify one of the common and obvious sign and symptom is portray of fever $(8,9)$. This implies that the majority of the participants may not have good knowledge about the signs and symptoms of dengue fever. Therefore, it is important to educate people on the signs and symptoms of dengue fever so as to seek immediate medical intervention in order to prevent unwarranted complications caused by dengue fever.

DENV NS1 antigenemia in relation to subject's clinical signs of illness showed no significant association $(\mathrm{P}>0.05)$. However, people with fever/ headache combination had highest cases of dengue virus NS1 antigenemia. Similar clinical manifestations have been reported in different studies $(10,11)$. This is of great public health importance because people who present with these symptoms at clinics and hos-
Table 1. Sociodemographic data of febrile subjects.

\begin{tabular}{lcc} 
Variables, observation & Frequency $($ tot=171) & $\%$ \\
Age, years (mean \pm SD) & $28.6 \pm 15.0$ & NA \\
Gender & 72 & \\
Male & 99 & 42.1 \\
Female & & 57.9 \\
\hline Residence & 153 & \\
Suburb & 18 & 89.5 \\
Village & & 10.5 \\
Educational level & 16 & \\
No formal education & 31 & 9.4 \\
Primary & 36 & 18.1 \\
Secondary & 33 & 21.1 \\
Undergraduate & 55 & 19.3 \\
Graduate & & 32.2 \\
\hline Occupation & 57 & \\
Civil servant & 9 & 33.3 \\
Driver & 6 & 5.3 \\
Farmer & 28 & 3.5 \\
Housewife & 23 & 16.4 \\
Unemployed & 48 & 13.5 \\
Other & & 28.1 \\
\hline
\end{tabular}

NA, Not available.

Table 2. Effects of preventive measures on Dengue virus NS1 antigenaemia among febrile Patients at UATH.

\begin{tabular}{|c|c|c|c|c|c|c|}
\hline Variables & $\begin{array}{c}\text { N. subjects } \\
\text { tested }\end{array}$ & $\begin{array}{l}\text { NS1 antigenaemia } \\
(\%)\end{array}$ & $\begin{array}{c}\text { Without NS1 } \\
\text { antigenaemia (\%) }\end{array}$ & P-value & RR & OR \\
\hline $\begin{array}{l}\text { Residence in proximity to refuse dumpsite }(<50 \text { meters }) \\
\text { Yes } \\
\text { No } \\
\text { Total }\end{array}$ & $\begin{array}{l}43 \\
128 \\
171\end{array}$ & $\begin{array}{l}13(30.2) \\
2(1.6) \\
15(8.8)\end{array}$ & $\begin{array}{l}30(69.7) \\
126(98.4) \\
156(91.2)\end{array}$ & $<0.0001^{*}$ & 0.9669 & 0.6462 \\
\hline $\begin{array}{l}\text { Frequent use of long sleeve clothing and trousers } \\
\text { Yes } \\
\text { No } \\
\text { Total }\end{array}$ & $\begin{array}{l}126 \\
45 \\
171\end{array}$ & $\begin{array}{l}6(4.8) \\
9(20.0) \\
15(8.8)\end{array}$ & $\begin{array}{l}120(95.2) \\
36(80.0) \\
156(91.2)\end{array}$ & $0.005^{*}$ & 0.9214 & 0.4500 \\
\hline $\begin{array}{l}\text { Consistent use indoor residual spraying } \\
\text { Yes } \\
\text { No } \\
\text { Total }\end{array}$ & $\begin{array}{l}142 \\
29 \\
171\end{array}$ & $\begin{array}{l}11(7.7) \\
4(13.8) \\
15(8.8)\end{array}$ & $\begin{array}{l}131(92.3) \\
25(86.2) \\
156(91.2)\end{array}$ & 0.491 & 0.9345 & 0.5248 \\
\hline $\begin{array}{l}\text { Presence of in-door water containers } \\
\text { Yes } \\
\text { No } \\
\text { Total }\end{array}$ & $\begin{array}{l}106 \\
65 \\
171\end{array}$ & $\begin{array}{c}11(10.4) \\
4(6.2) \\
15(8.8)\end{array}$ & $\begin{array}{l}95(89.6) \\
61(93.8) \\
156(100)\end{array}$ & 0.365 & 1.047 & 1.766 \\
\hline
\end{tabular}

Table 3. Distribution of Dengue virus NS1 antigenaemia across subjects' clinical signs of ill-health.

\begin{tabular}{lccc} 
Clinical signs & \multicolumn{2}{c}{ NS1 test results } & No of subjects tested (\%) \\
Fever and abdominal pain & $1(100)$ & $0(0.00$ & $1(100)$ \\
Fever alone & $57(90.5)$ & $6(9.5)$ & $63(100)$ \\
\hline Fever and headache & $27(84.4)$ & $5(15.6)$ & $32(100)$ \\
Fever, muscle and joint pains & $7(100.0)$ & $0(0.0)$ & $7(100)$ \\
\hline Fever and skin rash & $7(87.5)$ & $1(12.5)$ & $8(100)$ \\
$>2$ signs & $57(95.0)$ & $3(5.0)$ & $60(100)$ \\
\hline Total & $156(91.2)$ & $15(8.8)$ & $171(100)$
\end{tabular}


pitals are presumably treated for malaria especially in malaria endemic countries with limited diagnostic.

The ideal approach to prevent dengue is to eliminate areas where the vectors lays eggs. In this study, DENV NS1 antigenemia was significantly associated with residence in proximity to waste dumpsites; however it was not associated with the presence of indoor artificial water containers and consistent use of indoor residual spraying. Residence in proximity to waste dumpsites has been an environmental determinant and risk of Dengue virus infection. Vectors of Dengue virus in the urban cycle lay eggs in artificial water containers that could be found in many homes and waste dumpsites. Similar findings were reported by Siregar et al (12). Indiscriminate waste dumpsites and presence artificial water containers in residential areas encourages mosquito thriving/population and consequently places people who reside in communities to high risk of contracting Dengue virus (12).

With regards to the use of indoor residual spray, patients who had DENV NS1 antigenamia despite persistent use of indoor residual spraying could be due to insecticide-resistance exhibited by the mosquitoes. Perhaps, this could poses serious problem in the control of DENV transmission through chemical means (13). The use of insecticides in the control of the vector that transmit arboviruses is one of the preventive measures against associated diseases such as dengue and yellow fever (14). Pyrethroids such as deltamethrin, cypermethrin, and permethrin are being used to control adult Aedes mosquitoes (15).

The pyrethroids and DDT are widely used in Nigeria as indoor residual sprays and in Insecticide treated nets. Ae. aegypti has been reported to exhibit resistance to various these insecticides (16). This situation has created serious problems in vector control programs in Nigeria (17). However, only a few studies have investigated the insecticide susceptibility status of Ae. aegypti to permethrin and none to DDT (17).

The absence of association between DENV NS1 antigenaemia and presence of indoor water containers could be that larger water domestic containers for storing water were consistently covered, thus, limit the possibilities of being used as mosquitoes breeding sites. However, the presence of smaller water containers such as jars, used cans/plastic bottles/polythene bags may be unnoticed by residents.

\section{Conclusions}

Findings from this study imply that proper education and adherence to preventive measures minimize people from being susceptible to Dengue virus infections.

\section{References}

1. Ayukekbong JA. Dengue virus in Nigeria: current status and future perspective. Br J Virol 2014;1:106-11.
2. WHO Regional Office for South-East Asia. Comprehensive guidelines for prevention and control of dengue and dengue haemorrhagic fever, revised and expanded edition. New Delhi: World Health Organisation South East Asia Regional Office; 2011

3. World Health Organization. Global strategy for dengue prevention and control, 2012 2020. Geneva: WHO Press; 2012.

4. Madara AA, Abdulraheem NO. Relative abundance of adult mosquitoes in University of Abuja Main Campus, Abuja FCT, Nigeria. Nig J Parasitol 2013;34:1-5.

5. Thammapalo S, Chongsuvivatwong V, Geater A Dueravee M. Environmental factors and incidence of dengue fever and dengue haemorrhagic fever in an urban area, Southern Thailand. Epidemiol Infect 2008;136:135-43.

6. Siregar FA, Abdullah MR, Omar J, et al. Social and environmental determinants of dengue infection risk in North Sumatera Province, Indonesia. Asian J Epidemiol 2015;8:23-35.

7. Danbaba G, Nabegu AB, Binta A, Mustapha A. Assessment of implementation of the environmental sanitation policy in the federal capital territory (Fct) Abuja, Nigeria. Global J Soc Sci Stu 2016;2:1-13.

8. Chandren JR, Wong LP, AbuBakar S. Practices of dengue fever prevention and the associated factors among the Orang Asli in Peninsular Malaysia. PLoS Negl Trop Dis 2015;9:e0003954.

9. Shuaib F, Todd D, Campbell-Stennett DJ, Jolly PE. Knowledge, attitudes and practices regarding dengue infection in Westmoreland, Jamaica. West Indian Med J 2010;59:139-46.

10. Idris AN, Baba MM, Thairu Y, Bamidele O. Seroprevalence of Dengue type-3 virus among patients febrile illnesses attending a tertiary hospital in Maiduguri, Nigeria. Int J Med Med Sci 2013;5:560-3.

11. Idoko MO, Ado SA, Umoh VJ. Serological survey of dengue virus immunoglobulin $\mathrm{m}$ among febrile patients in Kaduna Metropolis, Nigeria. Aceh Int J Sci Tech 2014;3:152-8.

12. Siregar FA, Abdullah MR, Omar J, et al. Social and environmental determinants of dengue infection risk in north Sumatera province, Indonesia. Asian J Epidemiol 2015;8,23-35.

13. Vontas J, Kioulos E, Pavlidi N, et al. Insecticide resistance in the major dengue vectors Aedes albopictus and Aedes aegypti. Pest Biochem Physiol 2012;104:126-31.

14. Kamgang B, Marcombe S, Chandre F, et al. Insecticide susceptibility of Aedes aegypti and Aedes albopictus in Central Africa. Parasit Vectors 2011;4:79-87.

15. Macoris M, Andrighella M, Wanderley D, Ribolla P. Impact of insecticide resistance on the field control of Aedes aegypti in the state of Sao Paulo. Rev Soc Bras Med Trop 2014;47:573-8.

16. Koou SY, Chong CS, Vythilingam J, Lee C. Insecticide resistance and its underlying mechanisms in field populations of Aedes aegypti adults (Diptera: Culicidae) in Singapore. Parasit Vectors 2014;7:471

17. Kemabonta KA, Anikwe JC, Adaezeobiora I. Bioefficacy of skeetar in Anopheles gambiaeand Aedes aegypti mosquitoes from insecticides resistance areas in Lagos and Oyo State. Biol Agric Health Care 2013;3:122-35. 